\title{
Finding a Strong Stable Set or a Meyniel Obstruction in any Graph
}

\author{
Kathie Cameron ${ }^{1}$ and Jack Edmonds ${ }^{2}$ \\ ${ }^{1}$ Math Department, Wilfrid University, Waterloo, Ontario, Canada N2L 3C5, kcameron@wlu.ca \\ jackedmonds@rogers.com
}

\begin{abstract}
A strong stable set in a graph $G$ is a stable set that contains a vertex of every maximal clique of $G$. A Meyniel obstruction is an odd circuit with at least five vertices and at most one chord. Given a graph $G$ and a vertex $v$ of $G$, we give a polytime algorithm to find either a strong stable set containing $v$ or a Meyniel obstruction in $G$. This can then be used to find in any graph, a clique and colouring of the same size or a Meyniel obstruction.
\end{abstract}

Keywords: stable set, independent set, graph colouring, Meyniel graph, perfect graph

A Meyniel graph is a graph which does not contain an odd circuit with at least five vertices and at most one chord. Such a circuit is called a Meyniel obstruction. Meyniel [6] proved that Meyniel graphs are perfect. Meyniel's theorem can be stated in the following way.

Theorem 1 (Meyniel's Theorem) For any graph $G$, either $G$ contains a Meyniel obstruction, or $G$ contains a clique and colouring of the same size, or both.

We give a polytime algorithm to find, in any graph G, some instance of what Meyniel's Theorem says exists.

Burlet and Fonlupt [1] and Roussel and Rusu [7] gave polytime algorithms for recognizing whether or not a graph is a Meyniel graph. In the case that the graph is Meyniel, they do not find a clique and colouring of the same size. Our algorithm is incomparable with Meyniel graph recognition. It may give a clique and colouring the same size in a non-Meyniel graph without recognizing that the graph is nonMeyniel.

Algorithms for finding a minimum colouring of a Meyniel graph have been given by Hoàng [4], Hertz [3], Roussel and Rusu [8], and Lévêque and Maffray [5]. Any polytime algorithm for finding a minimum colouring in a perfect graph, in particular a Meyniel graph, can be used to find in polytime a clique in the graph which is the same size as the colouring [2,4]. However, none of these algorithms provide a way to find in any graph an instance of what Meyniel's Theorem asserts to exist. All of them, as well as ours, can be used to find a clique and colouring the same size in any graph which does not have a Meyniel obstruction. However our algorithm can also be described as finding a Meyniel obstruction in any graph which does not have a clique and colouring the same size.

A stable set in a graph $G$ is a set of vertices, no two of which are joined by an edge of $G$. A strong stable set is a stable set that contains a vertex of every maximal clique. (By maximal, we mean maximal 
with respect to inclusion, not largest.) It is easy to see that a polytime algorithm for finding a strong stable set in a graph can be applied repeatedly to find a colouring of a graph, and it is also then easy to construct a clique of the same size as the colouring.

Theorem 2 (Hoàng [4]) For any graph $G$ and vertex $w$ of $G$, either $G$ contains a strong stable set containing $w$, or $G$ contains a Meyniel obstruction, or both.

We give a polytime algorithm to find an instance of what Theorem 2 says exists. We now describe the ideas we use for developing this algorithm. As usual, we use $P_{4}$ to denote the path with four vertices.

A nice set $S$ is a maximal stable set linearly ordered so there is no induced $P_{4}$ between any vertex $u$ of $S$ and the pseudonode obtained by identifying all vertices of $S$ which precede $u$.

Theorem 3 Every nice set is a strong stable set.

Note that the definition of nice set is an NP description, but the definition of strong stable set is not.

\section{Algorithm.}

Input: Graph $G$ and vertex $w$ of $G$.

Output: Nice stable set of $G$ containing $w$ or a Meyniel obstruction.

Let $w=u_{1}$.

Suppose $u_{1}, u_{2}, \ldots, u_{k}$ have been chosen. If every vertex of $V(G)-\left\{u_{1}, u_{2}, \ldots, u_{k}\right\}$ is adjacent to one of $u_{1}, u_{2}, \ldots, u_{k}$, then the chosen vertices form a nice set. Otherwise, choose $u_{k+1}$ to be a vertex of $V(G)-\left\{u_{1}, u_{2}, \ldots, u_{k}\right\}$ not adjacent to any of $u_{1}, u_{2}, \ldots, u_{k}$ and such that it has the largest number of common neighbours with the pseudonode $v\left(u_{1}, u_{2}, \ldots, u_{k}\right)$ obtained by identifying $u_{1}, u_{2}, \ldots, u_{k}$. If there is a $P_{4}$ from $v\left(u_{1}, u_{2}, \ldots, u_{k}\right)$ to $u_{k+1}$, then $G$ contains a Meyniel obstruction. To find this circuit, we use a "pseudonode expansion algorithm", which we cannot describe here. The simple lemmas below help us to find the circuit.

A chord of a circuit $C$ is called short if it joins two vertices at distance 2 in $C$ (i.e., if it creates a triangle with $C$ ). Two short chords of $C$ are overlapping if one is $a c$ and the other is $b d$, where $a, b, c, d$ are consecutive vertices on $C$.

Lemma 1 In an odd circuit of size at least 7 with two chords, either there is an odd circuit of size at least 5 with at most one chord, or the two chords are overlapping short chords..

Lemma 2 In an odd circuit of size at least 5 with all chords hitting the same vertex $h$ and at least one of these possible chords missing, there is an odd circuit of size at least 5 with at most one chord, and the chord is short and hits $h$.

\section{References}

[1] M. Burlet and J. Fonlupt, Polynomial algorithm to recognize a Meyniel graph, Topics on Perfect Graphs, 225-252, North-Holland Math. Stud., 88, North-Holland, Amsterdam, 1984.

[2] M. Grötschel, L. Lovász and A. Schrijver, Polynomial algorithms for perfect graphs, Topics on Perfect Graphs, 325-356, North-Holland Math. Stud., 88, North-Holland, Amsterdam, 1984. 
[3] A. Hertz, A fast algorithm for coloring Meyniel graphs. J. Combin. Theory Ser. B 50 (1990), 231240.

[4] C. T. Hoàng, On a conjecture of Meyniel, J. Combin. Theory Ser. B 42 (1987), 302-312.

[5] B. Lévêque abd F. Maffray, Coloring Meyniel graphs in linear time, preprint, 2004.

[6] H. Meyniel, The graphs whose odd cycles have at least two chords, Topics on Perfect Graphs, 115119, North-Holland Math. Stud., 88, North-Holland, Amsterdam, 1984.

[7] R. Roussel and I. Rusu, Holes and dominoes in Meyniel graphs, Int. J. Found. Computer. Sci. 10 (1999), 127-146.

[8] F.Roussel and I. Rusu, An $O\left(n^{2}\right)$ algorithm to color Meyniel graphs, Discrete Math. 235 (2001), 107-123. 
